Check for updates

Cite this: RSC Adv., 2019, 9, 10049

\title{
Synthesis and properties of castor oil based plasticizers
}

\author{
Qinghe Fu, ${ }^{a}$ Yilang Long, ${ }^{b}$ Yingyun Gao, ${ }^{a}$ Yuan Ling, ${ }^{a}$ Hao Qian, ${ }^{a}$ Fang Wang ${ }^{\star a}$ \\ and Xinbao Zhu (D)*ab
}

A series of environment-friendly plasticizers has been synthesized from castor oil through a mild esterification/epoxidation reaction. The modified epoxy acetylated castor oil (EACO) can plastify poly(vinyl chloride) (PVC) efficiently, even better than the commercial plasticizers dioctyl terephthalate (DOTP) and epoxidized soybean oil (ESO), in terms of in tensile strength, migration stability, solvent extraction stability and thermal stability. Specifically, the tensile strength and elongation at break of a PVC sample plastified by epoxy acetylated castor oil (EACO) were 18.5 and $10.0 \%$ higher than that of DOTP, and 13.9 and $23.8 \%$ higher than that of ESO, respectively. Volatility, migration, solvent extraction and thermal stability tests indicated that the presence of carbon-carbon double bonds and hydroxy groups reduce the compatibility of a plasticizer with PVC while the presence of epoxy groups and ester bonds can improve the plasticizing effect of the plasticizer on PVC. In addition, alkyl groups can improve the plasticizing effect on PVC while benzene rings increase the rigidity of the PVC. The design strategy based on castor oil highlights a sustainable avenue for preparing cost-effective and high-efficiency plasticizers.

Received 15th December 2018

Accepted 18th March 2019

DOI: $10.1039 / c 8 r a 10288 k$

rsc.li/rsc-advances a role as stabilizer. ${ }^{10}$ When heated, PVC undergoes autocatalytic dehydrochlorination, whereas the epoxide group can scavenge the produced $\mathrm{HCl}$ which would have been constantly generated by a free radical reaction from PVC through induced catalytic degradation. ${ }^{\mathbf{1 1}}$ Currently, an epoxy fatty acid multi-ester, ${ }^{\mathbf{1 2}}$ dimer acid ester ${ }^{13}$ and fatty acid glycidyl ester ${ }^{14}$ are also used as plasticizers in PVC. However, most of these plasticizers, which are based on vegetable oils still have a problem in terms of their also being edible food ingredients.

Castor oil, a land-grown oil, is an inedible oil and a highly renewable resource for many chemical industries, and is mainly produced in Asia and Africa. Castor oil molecules contain hydroxy groups, carbon-carbon double bonds and ester bonds, which can be undergo epoxidation, sulfonation, halogenation, esterification, hydrolysis and other reactions to produce different varieties of plasticizers. For example, epoxidized methyl acetoricinoleate is a common castor oil based plasticizer with high epoxy value and good thermal stability, ${ }^{15}$ but a large amount of glycerol is produced during its preparation, which represents excess capacity. Two types of epoxy acetyl castor oil polyol ester plasticizers were synthesized by Jia et al. and had better plasticizing effect than DOP and ESO ${ }^{16}$ but the tensile strength of the PVC blends was much lower than that from ESO, which makes its replacing of soybean oil impractical. A castor oil-based diglycidyl ester plasticizer (C26-DGE) was prepared by Chen et al. ${ }^{17}$ with ricinoleic acid as raw material and results showed that the plasticizer endowed the PVC matrix with enhanced compatibility and flexibility. Besides, a series of
${ }^{a}$ College of Chemical Engineering, Nanjing Forestry University, Nanjing 210037, China. E-mail: zhuxinbao@njfu.com.cn

${ }^{b}$ Anhui Engineering Research Center of Epoxy Resin and Additives, Huangshan 245900, China 
phosphorus-containing flame retardant plasticizers based on castor oil as a secondary plasticizer were synthesized and the results showed that the material added plasticizers had improved tensile strength, with good heat insulation and flame retardant properties, but the elongation at break was decreased. ${ }^{18-21}$

Our team has conducted a substantial amount of work on the synthesis of plasticizers, especially in clean production processes. ${ }^{22-24}$ In this study, we synthesized five environmentfriendly plasticizers derived from castor oil through mild esterification/epoxidation reactions. The synthetic plasticizers were added to PVC (along with a heat stabilizer), and the properties were investigated and compared to the commercial plasticizers DOTP and ESO. We compared the effects of epoxy groups and carbon-carbon double bonds, ester groups and hydroxyl groups, and alkyl and benzene rings on plasticizing PVC to attempt to find an environment-friendly plasticizer with good plasticity ability using a simple synthesis method. We expect more competitive results compared with traditional plasticizers to lead the way towards green and sustainable routes for PVC application.

\section{Experimental section}

\section{Materials}

Hydrogen peroxide, acetic anhydride, benzoyl chloride, acetic acid, calcium oxide, toluene, sodium hydroxide, sodium carbonate, hydrochloric acid and acetone were provided by Nanjing Chemical Reagent Co., Ltd. (China); castor oil and the cation exchange resin were provided by Anhui Xinyuan Chemical Co., Ltd. (China).

\section{Synthesis of epoxy castor oil (ECO)}

Castor oil (93.4 g), acetic acid (18.0 g), toluene $(100 \mathrm{~mL})$ and cation exchange resin catalyst $(4.7 \mathrm{~g})$ were added to a $500 \mathrm{~mL}$ four-necked flask equipped with a stirrer, a thermometer, a constant pressure funnel and a ball condenser, 30\% hydrogen peroxide (39.7 $\mathrm{g}$ ) was dropwise added in the reaction mixture over $2 \mathrm{~h}$ and stirred at $60{ }^{\circ} \mathrm{C}$ for $5 \mathrm{~h}$. Then the reaction mixture was filtered to recycle the cation exchange resin, and the remaining fluid was washed to $\mathrm{pH}=7$ with sodium carbonate solution and distilled water. Then the water and solvent were removed by vacuum distillation. Scheme 1 shows the synthesis routes of all products.

\section{Synthesis of acetylated castor oil (ACO)}

Castor oil (93.4 g) and acetic anhydride (27.5 g) were added to a $500 \mathrm{~mL}$ four-necked flask, and were stirred at $140{ }^{\circ} \mathrm{C}$ for $2 \mathrm{~h}$. Then the reaction mixture was washed to $\mathrm{pH}=7$ with sodium carbonate solution and distilled water. Then the water was removed by vacuum distillation.

\section{Synthesis of epoxy acetylated castor oil (EACO)}

Castor oil (93.4 g) and acetic anhydride (27.5 g) were added to a $500 \mathrm{~mL}$ four-necked flask and were stirred at $140{ }^{\circ} \mathrm{C}$ for $2 \mathrm{~h}$. Then the reaction mixture was cooled to $60{ }^{\circ} \mathrm{C}$, and toluene $(100$
$\mathrm{mL}$ ) and cation exchange resin catalyst ( $4.7 \mathrm{~g})$ were added, after that $30 \%$ hydrogen peroxide $(39.7 \mathrm{~g}$ ) was added dropwise to the reaction mixture over $2 \mathrm{~h}$ and then stirred for $5 \mathrm{~h}$. Then the reaction mixture was filtered to recycle the cation exchange resin, and the remaining fluid was washed to $\mathrm{pH}=7$ with sodium carbonate solution and distilled water. Then the water and solvent were removed by vacuum distillation.

\section{Synthesis of benzoyl castor oil (BCO)}

Castor oil (93.4 g), benzoyl chloride (38.0 g), calcium oxide (10.6 $\mathrm{g})$ and toluene $(100 \mathrm{~mL})$ were added to a $500 \mathrm{~mL}$ four-necked flask and were stirred at $110{ }^{\circ} \mathrm{C}$ for $3 \mathrm{~h}$. Then the reaction mixture was washed to $\mathrm{pH}=7$ with sodium hydroxide solution and distilled water. Then the water and solvent were removed by vacuum distillation.

\section{Synthesis of epoxy benzoyl castor oil (EBCO)}

BCO (121.5 g), acetic acid (18.0 g), toluene $(100 \mathrm{~mL})$ and cation exchange resin catalyst $(4.7 \mathrm{~g})$ were added to a $500 \mathrm{~mL}$ fournecked flask and were heated and 30\% hydrogen peroxide (39.7 g) was added dropwise to the reaction mixture over $2 \mathrm{~h}$ and stirred at $60{ }^{\circ} \mathrm{C}$ for $5 \mathrm{~h}$. Then the reaction mixture was filtered to recycle the cation exchange resin, and the remaining fluid was washed to $\mathrm{pH}=7$ with sodium carbonate solution and distilled water. Then the water and toluene were removed by vacuum distillation.

\section{Preparation of plasticized PVC samples}

The PVC, plasticizer and heat stabilizer were mixed in the ratio of $50: 25: 1$. The mixture was blended for $7 \mathrm{~min}$ at $170{ }^{\circ} \mathrm{C}$ using a HAAKE PolyLab OS torque rheometer, and then placed in a flat-panel curing machine and pressed for $4 \mathrm{~min}$ at $170{ }^{\circ} \mathrm{C}$ and $2 \mathrm{~min}$ at room temperature. The PVC blends were labeled as PVC-DOTP (PVC/DOTP), PVC-ESO (PVC/ESO), PVC-ECO (PVC/ ECO), PVC-ACO (PVC/ACO), PVC-EACO (PVC/EACO), PVC-BCO (PVC/BCO) and PVC-EBCO (PVC/EBCO).

\section{Characterizations and measurements}

FT-IR spectra were recorded on a Nicolet FTIR-360 (Nicolet Instrument Corp., USA) Fourier transform infrared spectrophotometer. The spectra was acquired in the range of 400 to $4000 \mathrm{~cm}^{-1}$.

${ }^{1} \mathrm{H}$ NMR spectra were recorded by using an AVANE400 (Bruker Company, Switzerland) with deuterated chloroform as a solvent.

Tensile strength and elongation at break of all PVC samples were determined according to the GB/T 1040.1-2006 (China) under ambient conditions by using an E43.104 Universal Testing Machine (MTS Instrument Corp., China).

The volatility stability tests were determined by GB/T 38302008, PVC samples were cut into $50 \mathrm{~mm} \times 50 \mathrm{~mm}$ squares, placed in an oven for $24 \mathrm{~h}$ at $80{ }^{\circ} \mathrm{C}$, and then cooled to room temperature in a desiccator. The mass changes were measured by weighing before and after heating. The volatility rate was 


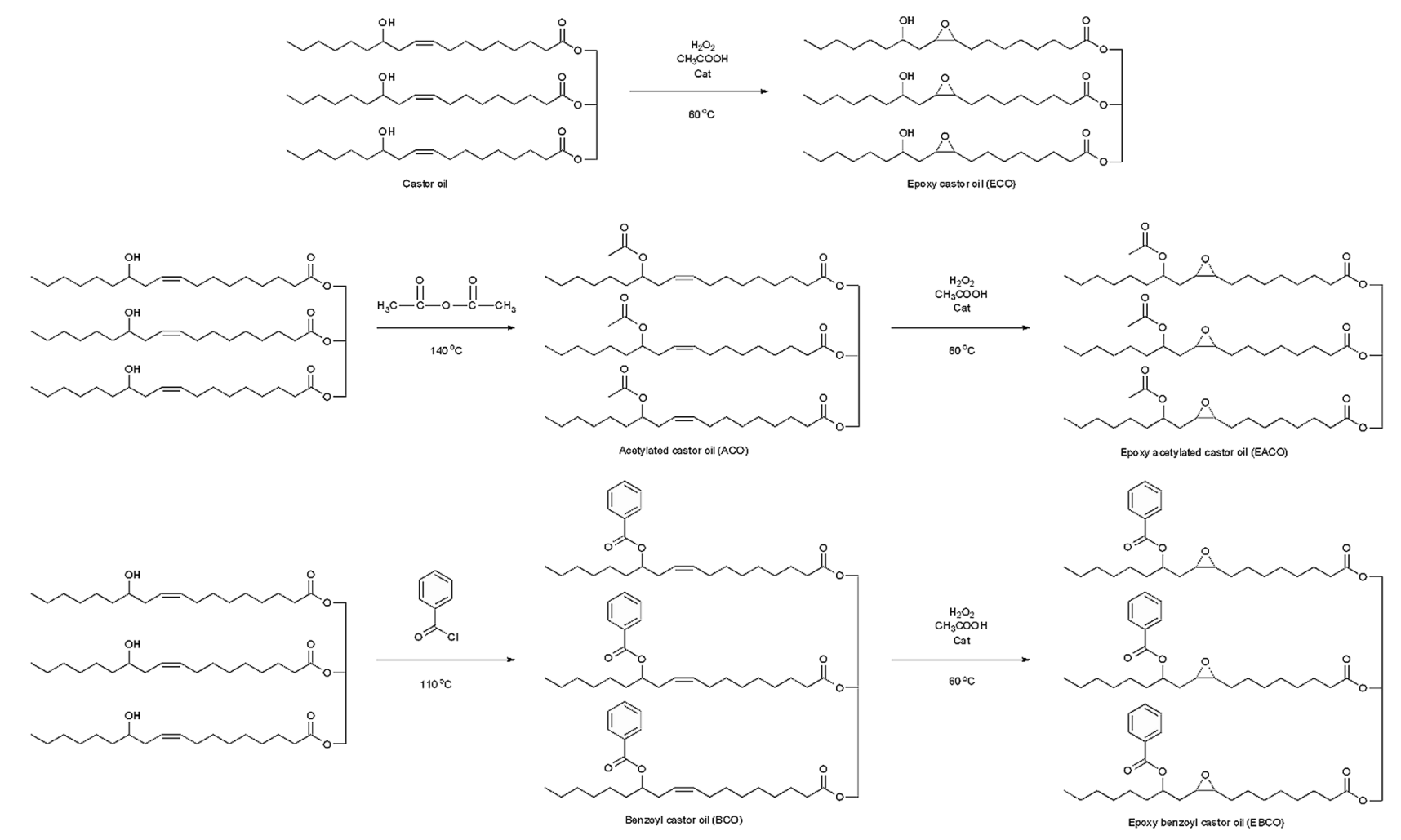

Scheme 1 Synthesis of ECO, ACO, EACO, BCO and EBCO.

calculated as the ratio of the evaporated and the initial plasticizer.

Migration stability tests were determined according to the ISO 176-2005, PVC samples were cut into $50 \mathrm{~mm} \times 50 \mathrm{~mm}$ squares, and covered with activated carbon on both sides. Samples were placed in an oven for $24 \mathrm{~h}$ at 20 and $70{ }^{\circ} \mathrm{C}$, and weight loss was measured before and after heating.

The solvent extraction stability tests were determined according to the GB/T3830-2008, PVC samples with $50 \mathrm{~mm} \times$ $50 \mathrm{~mm}$ shape were immersed in five different solvents (distilled water, $30 \%(\mathrm{w} / \mathrm{w})$ acetic acid, $30 \%(\mathrm{w} / \mathrm{w})$ sodium hydroxide, $50 \%$ $(\mathrm{w} / \mathrm{w})$ ethanol and cyclohexane) for $24 \mathrm{~h}$ at room temperature. Subsequently, samples were placed in an oven for $8 \mathrm{~h}$ at $50{ }^{\circ} \mathrm{C}$ and cooled to room temperature in a desiccator. The mass rate changes were measured before and after these processes.

The dynamic mechanical analyses were performed via a DMTA Q800 (TA Instruments, US) using rectangular samples of geometry $35 \mathrm{~mm}(L) \times 12.5 \mathrm{~mm}(W) \times 3.2 \mathrm{~mm}(T)$. The oscillatory frequency of the dynamic tests were $1 \mathrm{~Hz}$. The temperature was raised at a rate of $3{ }^{\circ} \mathrm{C} \mathrm{min}^{-1}$ from -60 to $+120{ }^{\circ} \mathrm{C}$.

The thermal ability of PVC blends was characterized in a DTG-60AH TGA thermal analysis instruments set-up (Netzsch Instrument Crop., Germany) in an $\mathrm{N}_{2}$ atmosphere (50 $\mathrm{mL} \min ^{-1}$ ) at a heating rate of $10{ }^{\circ} \mathrm{C} \mathrm{min}^{-1} .5 \mathrm{mg}$ of samples were placed into platinum pans and scanned from 30 to $600^{\circ} \mathrm{C}$.

The fracture surface morphology of the PVC blends after tensile fracture were analysed by a Hitachi S-4800 (Hitachi, Japan) scanning electron microscope (SEM) operated at $1 \mathrm{kV}$.

\section{Results and discussion}

\section{FTIR and NMR characterization of the synthesized plasticizers}

The FT-IR spectra of CO, ECO, ACO, EACO, BCO and EBCO are shown in Fig. 1. Compared to CO and ECO, the hydroxy group signal at $3400 \mathrm{~cm}^{-1}$ almost disappeared in the spectra of ACO, EACO, BCO and EBCO, which indicated that acylation reaction occurred. The epoxy group at $1068 \mathrm{~cm}^{-1}$ appeared in the FT-IR of ECO, EACO and EBCO, while the carbon-carbon double bonds of $\mathrm{CO}, \mathrm{ACO}$ and $\mathrm{BCO}$ at around $3010 \mathrm{~cm}^{-1}$ disappeared, which demonstrated that the carbon-carbon double bonds have been converted into epoxy groups.

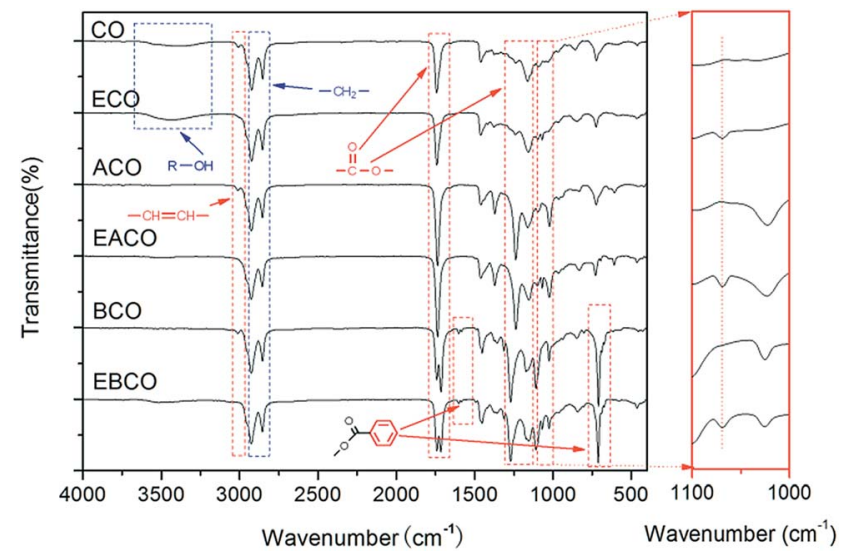

Fig. 1 FT-IR spectra of $\mathrm{CO}$ and the synthesized plasticizers. 
Fig. 2 shows the ${ }^{1} \mathrm{H}$ NMR spectra of CO, ECO, ACO, EACO, $\mathrm{BCO}$ and EBCO. Compared to the $\mathrm{CO}{ }^{1} \mathrm{H}$ NMR spectra, the proton signals in the 5.45-5.55 ppm region of ECO, EACO and EBCO spectra associated with carbon-carbon double bond bands were weakened, and signals in the 2.95-3.15 ppm region of ECO, EACO and EBCO spectra associated with epoxy groups bands indicated that during the epoxidation reaction, most of the carbon-carbon double bonds have been converted. The signals in the $2.01 \mathrm{ppm}$ region of ACO and EACO spectra are associated with shifted methyl hydrogens of the acetyl group, so verifying the acetylation reaction. Also, the proton signals in the 7.45-7.55 and 8.1 ppm region of BCO and EBCO spectra associated with benzene revealed that the hydroxy group of castor oil was acylated with benzoyl chloride.

\section{Tensile test}

The results of tensile strength and elongation at break are presented in Fig. 3. PVC-ECO shows a lower tensile strength and lower elongation compared with the other six samples. After acetylation or benzoylation of hydroxy groups and subsequent epoxidation, there is an obvious increase in tensile strength and elongation of PVC-EACO and PVC-EBCO, which indicated that the hydroxy group can reduce the compatibility of plasticizers with PVC. ${ }^{15}$ Compared to PVC-ACO and PVC-BCO, PVC-EACO and PVC-EBCO displayed superior tensile strength and break elongation, which can be attributed to that the epoxidation of carbon-carbon double bonds enhanced the plasticizer so as to be compatible with PVC. ${ }^{25,26}$ The tensile strength and elongation at break of PVC-EACO were 18.5 and $10.0 \%$ higher than PVC-DOTP, and 13.9 and $23.8 \%$ higher than PVC-ESO, respectively, demonstrating that the plasticizing effect of EACO on PVC is more efficient than that of DOTP and ESO. This may be because EACO has more epoxy groups than DOTP and more ester bonds than ESO, which can obviously increase the compatibility of plasticizers and PVC. ${ }^{14}$ PVC-EBCO showed the highest tensile strength (a) $\mathrm{CO}$

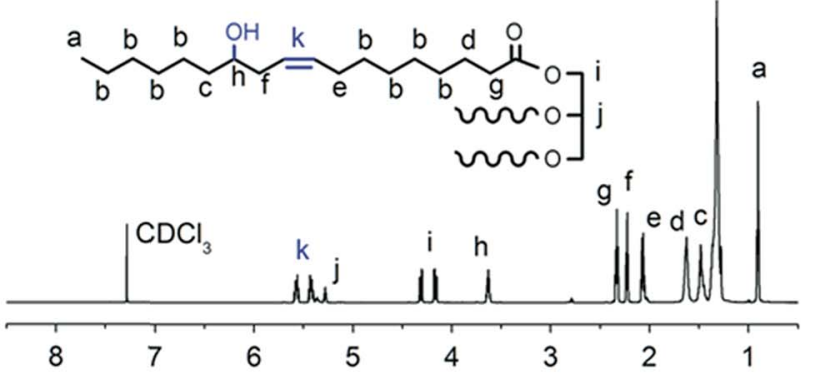

(c) ACO
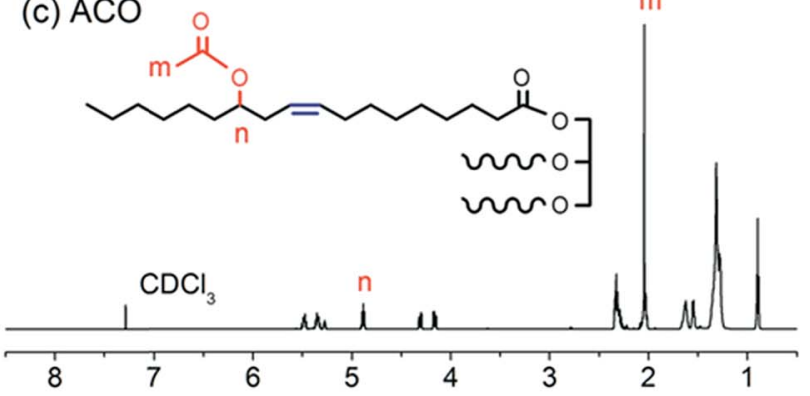

(e) $\mathrm{BCO}$

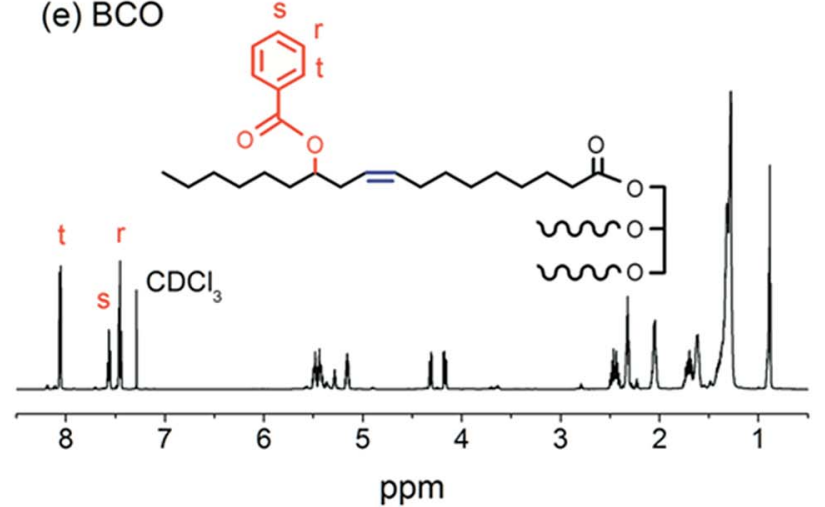

(b) ECO

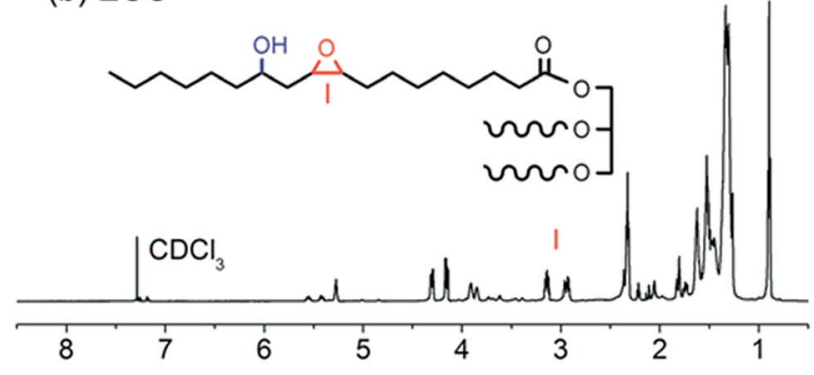

(d) EACO
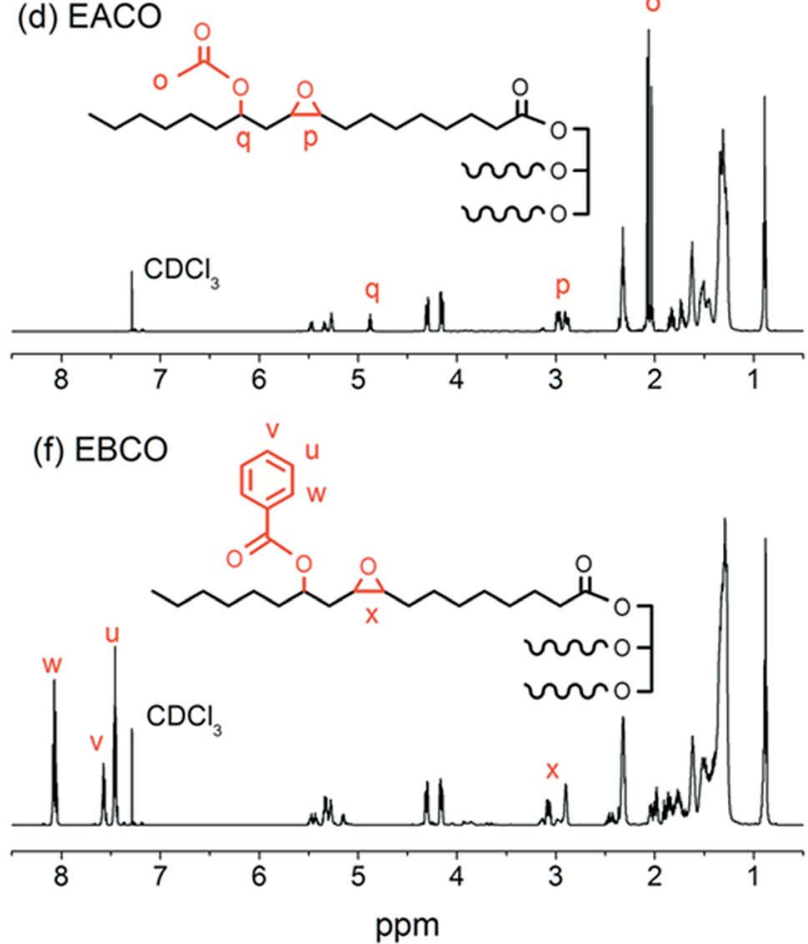

Fig. $2{ }^{1} \mathrm{H}$ NMR spectrum of (a) CO, (b) ECO, (c) ACO, (d) EACO, (e) BCO and (f) EBCO. Note: ' $\Omega$ $\Omega$ ' represents the same structure and functional group as the first molecular chain in their respective chemical structures. 


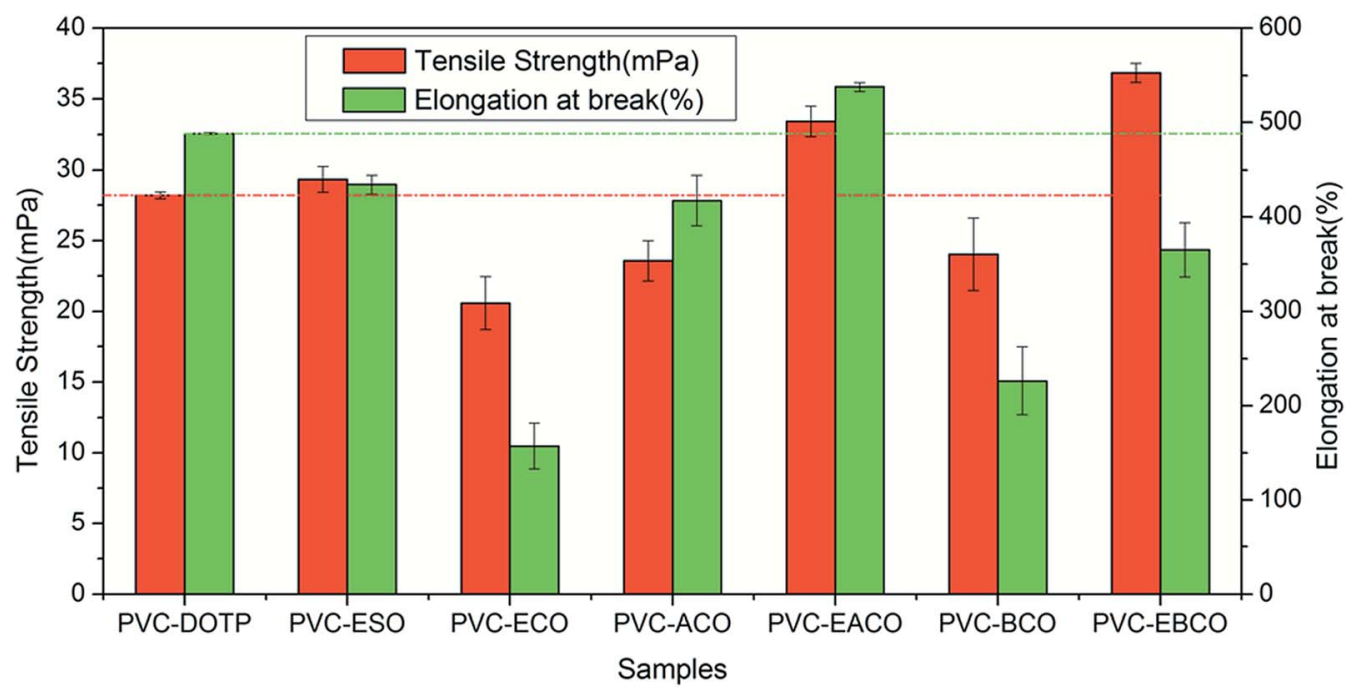

Fig. 3 Tensile properties of the PVC blends.

compared with the other six samples and lower elongation than PVC-DOTP, PVC-ESO, PVC-ACO and PVC-EACO, which meant that the rigid benzene ring on the plasticizer structure can improve the tensile strength of the blend and reduce the fluidity of the plasticizer in PVC. ${ }^{77}$ Plasticizer EBCO can thus be used in materials requiring high rigidity.

\section{Volatility stability test}

The volatility stability of all plasticizers was investigated, and the results are shown in Fig. 4. PVC-ECO shows the highest volatility, which proved that the plasticizer ECO exhibited worst compatibility towards PVC owing to the presence of the hydroxyl group. The lower mass change of PVC-EACO and PVC-EBCO when compared to PVC-ACO and PVC-BCO indicated that the epoxide functional groups of EACO and EBCO play a more significant role than the double bonds of ACO and BCO. Additionally, the mass change of PVC films plastified by acetylationmodified plasticizers is less than that upon benzoylation modification, which illustrated that alkyl groups can increase the compatibility of plasticizers and PVC more than benzene rings.

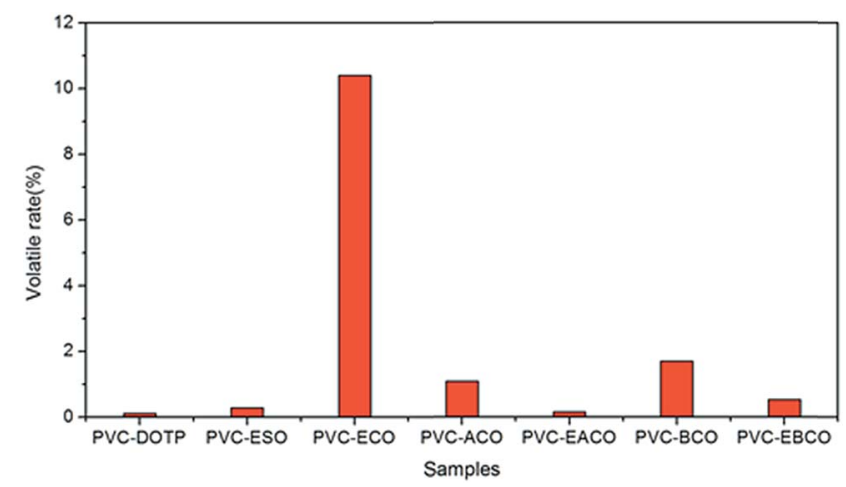

Fig. 4 Volatility properties of the PVC blends.

\section{Migration stability test}

The migration stability of the different plasticizers in the PVC blends is shown in Fig. 5 . At $20^{\circ} \mathrm{C}$, the weight loss of all samples is small, on account of the low molecular mobility at low temperature. At $70{ }^{\circ} \mathrm{C}$, the trend is similar to that of volatility stability. The results further illustrate that the hydroxy group reduced the compatibility of plasticizers with PVC while the epoxy group could improve the volatility and migration stability of the plasticizer.

\section{Migration stability test}

The solvent extraction stability of the different plasticizers in PVC blends is shown in Fig. 6. The weight loss of PVC blends is strongly affected by the strength of the interactions between the plasticizers and the polymer chain..$^{28}$ PVC-ECO, PVC-ACO and PVC-BCO show higher dissolution rate than PVC-DOTP, PVCESO and PVC-EACO in distilled water, $30 \%(\mathrm{w} / \mathrm{w})$ acetic acid, $30 \%(\mathrm{w} / \mathrm{w})$ sodium hydroxide and $50 \%(\mathrm{w} / \mathrm{w})$ ethanol, which indicate that the hydroxy group and the double bonds have a negative effect on the interactions between the plasticizers

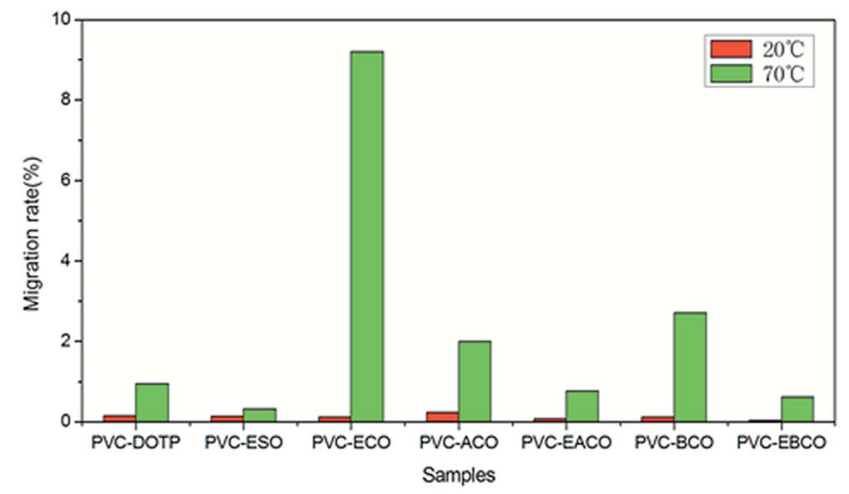

Fig. 5 Migration stabilities of the PVC blends. 


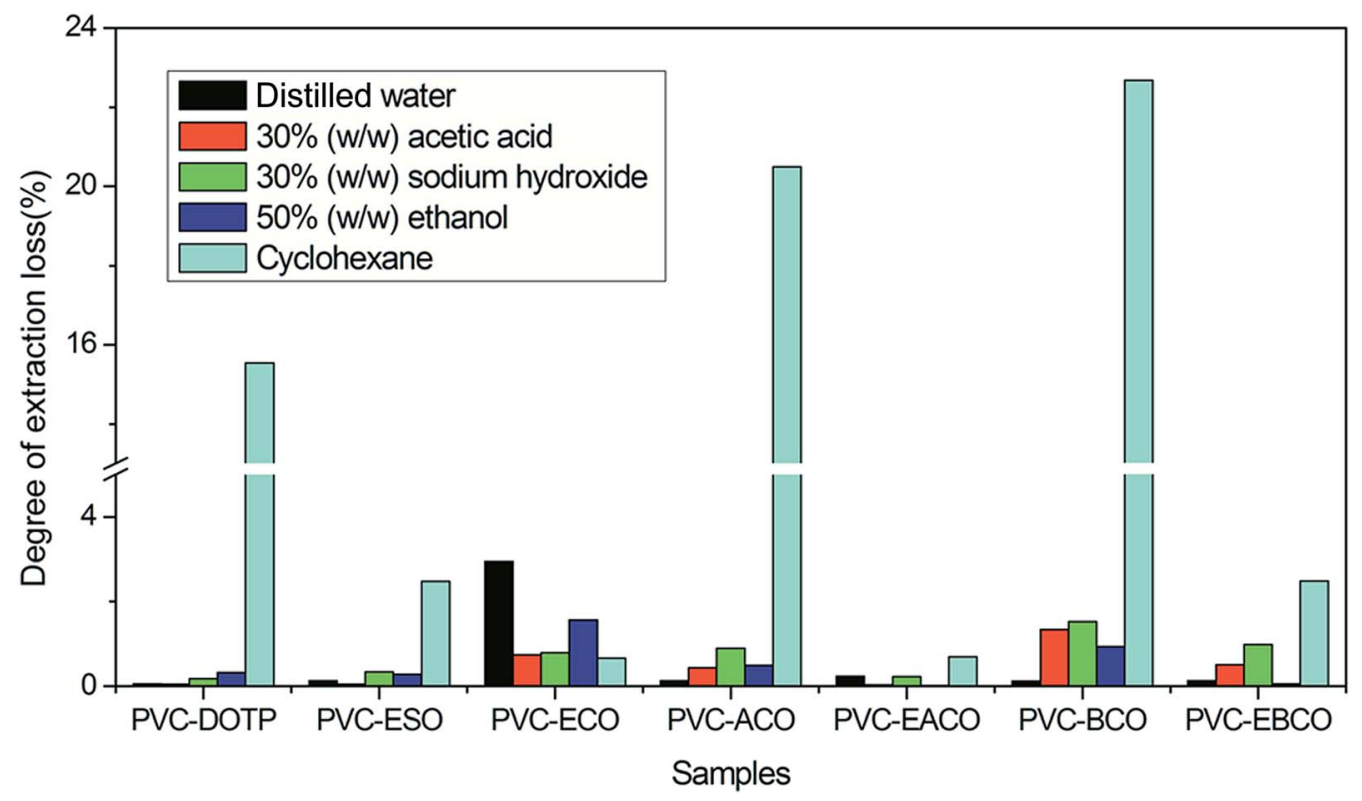

Fig. 6 Solvent extraction stabilities of the PVC blends.

and PVC, which made more plasticizer transfer to the solvent from PVC. However, in cyclohexane, PVC-ESO, PVC-ECO, PVCEACO and PVC-EBCO have a lower extraction rate than the other three samples, which may be because the epoxy group is a polar group, and has a stronger interaction with the polar parts of the PVC molecules, which reduces the plasticizer dissolution rate. ${ }^{27}$ Moreover, PVC-EBCO presents a higher dissolution rate compared with PVC-EACO, even though EBCO has no hydroxy groups or carbon-carbon double bonds. However, the non-polar benzene rings of EBCO serve to weaken the attractive forces between the plasticizer and PVC chains, thus increasing the free volume and imparting flexibility to the polymer. ${ }^{29}$ On the whole, PVC-EACO had better solvent extraction stability than PVC-DOTP and PVC-ESO, which signifies that EACO has higher potential application value in food packing and medical devices.

\section{Dynamic mechanical property test}

According to Fig. 7, the storage modulus of PVC-EACO is higher than that of PVC-DOTP and PVC-ESO, which indicated that PVCEACO has a higher elasticity. At the same time, the acetylation and epoxidation modified PVC test pieces had higher storage modulus than for benzoylation and non-epoxidation samples, demonstrating that the alkyl and epoxy groups enhance the PVC elasticity compared to the benzene ring and carbon-carbon double bonds.

The loss modulus (Fig. 8), which represents the amount of energy lost by viscous deformation of the material, is characterized by a peak value as well as the curve of the damping factor ( $\tan \delta$, Fig. 9), and generally the peak value of $T$ is taken as the glass transition temperature. In most cases, a narrow $\tan \delta$ peak and single $T_{\mathrm{g}}$ of the polymer composite indicate excellent homogeneity. ${ }^{26}$ PVC-DOTP, PVC-ESO, PVC-ACO, PVC-EACO,
PVC-BCO and PVC-EBCO showed only one $\tan \delta$ peak, and the $T_{\mathrm{g}}$ values were $35.2,39.8,70.2,35.7,76.9$ and $60.1{ }^{\circ} \mathrm{C}$ respectively, indicating that the six plasticizers were compatible with PVC, while ECO was not, with two tan $\delta$ peaks at -34.8 and 86. ${ }^{\circ} \mathrm{C}$ in PVC-ECO. PVC-EACO and PVC-EBCO show a lower $T_{\mathrm{g}}$ value than PVC-ACO and PVC-EBCO respectively, which demonstrates that the epoxy groups can make the molecule more polar, and hydrogen bonds formed between the molecular chains can ultimately increase the viscous activity of the mixture. $^{30}$

\section{Thermogravimetric analysis}

The TGA of PVC plasticized with different plasticizers is presented in Fig. 10. From the TGA curves of PVC blends, it can be

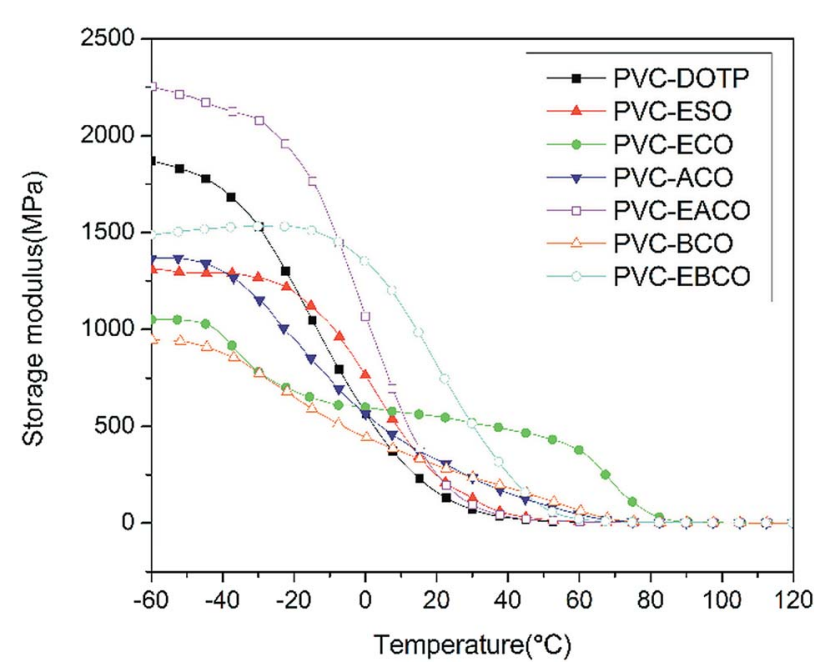

Fig. 7 Storage modulus of the PVC blends. 


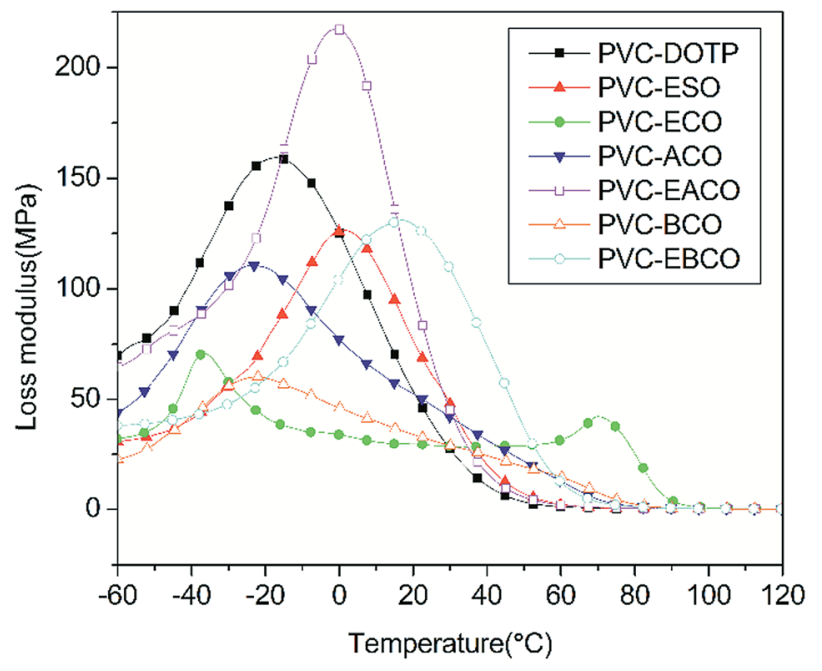

Fig. 8 Loss modulus of the PVC blends.

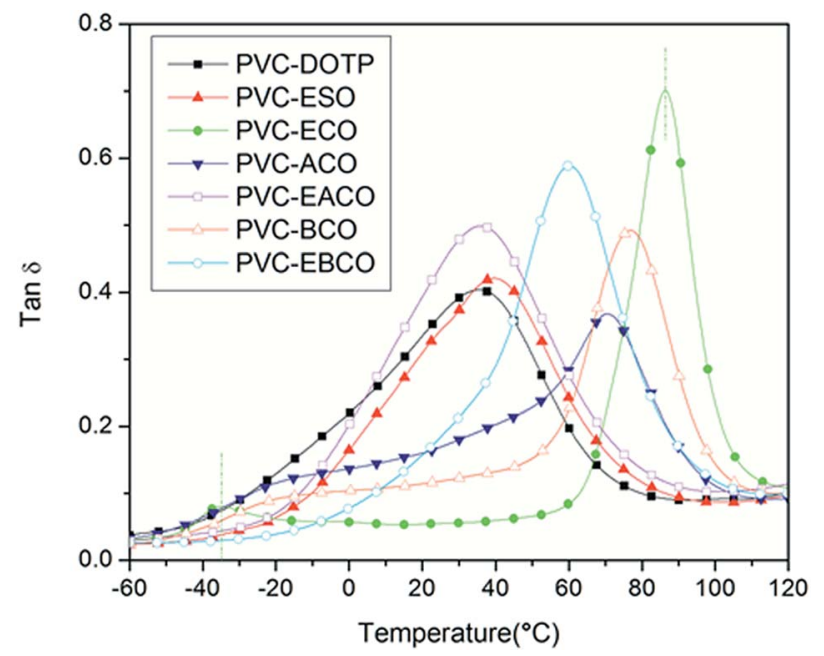

Fig. 9 Loss tangent curves as a function of temperature of the PVC blends.

seen that all of the PVC blends were thermally stable in a nitrogen atmosphere below $220^{\circ} \mathrm{C}$ and exhibited a two-stage thermal degradation process above that temperature. The first degradation at about $260-350{ }^{\circ} \mathrm{C}$ corresponds to the elimination of a large amount of $\mathrm{HCl}$. The second stage at about $440-$ $480{ }^{\circ} \mathrm{C}$ is attributed to the decomposition of the PVC main chain and plasticizers. The thermal performance data including the weight loss of $10 \%\left(T_{10 \%}\right)$, the weight loss of $50 \%$ $\left(T_{50 \%}\right)$ and the maximum weight-loss temperature rate $\left(T_{\mathrm{P} 1}\right.$ and $T_{\mathrm{P} 2}$ ) are summarized in Table $1 . T_{10 \%}, T_{50 \%}$ and $T_{\mathrm{P} 1}$ values of PVC plasticized with ESO, ECO, ACO, EACO, BCO and EBCO were higher than for DOTP, which may be because compared to DOTP, the other six plasticizers have more ester bond structures and epoxy groups, which are associated with higher thermal stability than the lower-thermally stable chain segments of DOTP, thereby increasing the thermal stability of the PVC blends. ${ }^{16}$ The $T_{\mathrm{P} 1}$ values of PVC plasticized with ESO,

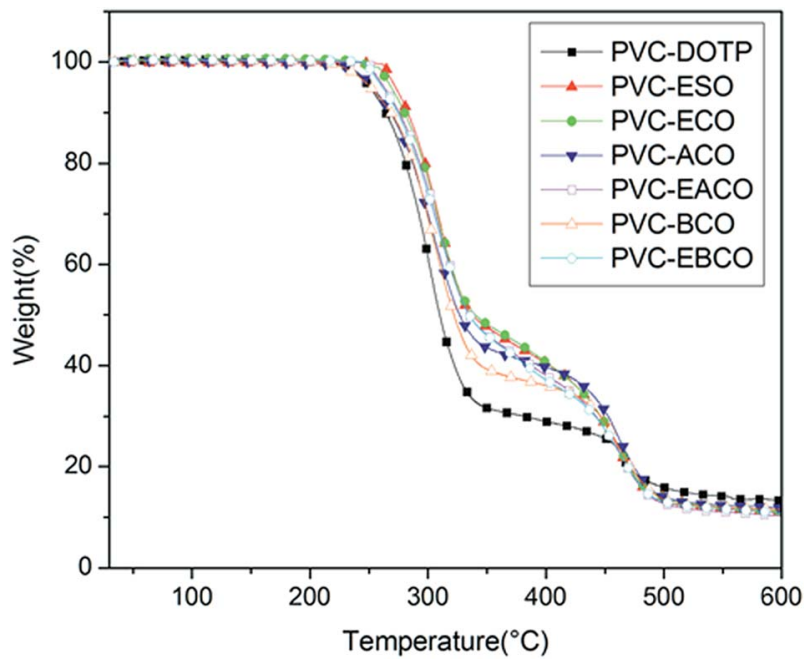

Fig. 10 TGA curves of the PVC blends.

ECO, EACO and EBCO were higher than those with DOTP, ACO and $\mathrm{BCO}$, which proves that epoxy groups can absorb hydrogen chloride degraded by light and heat, preventing the continuous decomposition of PVC and prolonging the lifetime of PVC. ${ }^{31,32}$ In addition, the $T_{\mathrm{P} 2}$ values of PVC-ACO and PVC-BCO are lower than those of PVC-EACO and PVC-EBCO, which may be because the double bonds in $\mathrm{ACO}$ and $\mathrm{BCO}$ reduced the thermal stability. The char residue of PVC plasticized with DOTP is higher than that of the other plasticizers, indicating that ESO, ECO, ACO, EACO, BCO and EBCO could decrease the char residue of PVC blends more than DOTP. From these data, we could conclude that the castor oil based plasticizers could improve the thermal stability and reduce the amount of char residue of PVC blends.

\section{Fracture surface morphology analysis}

The fracture surface morphology of pulled and cut plasticized PVC blends is shown in Fig. 11. As can be seen, the section topography of the different PVC blends is quite different. PVCDOTP, PVC-ESO, PVC-ACO, PVC-EACO, PVC-BCO and PVCEBCO clearly showed numerous small protrusions and nonretracted filaments, which are typical features of ductile fracture, indicating that the plasticizers have great compatibility with PVC and a good plasticizing effect. However, PVC-

Table 1 TGA data of the PVC blends

\begin{tabular}{lccccc} 
Samples & $T_{10 \%} /{ }^{\circ} \mathrm{C}$ & $T_{50 \%} /{ }^{\circ} \mathrm{C}$ & $T_{\mathrm{P} 1} /{ }^{\circ} \mathrm{C}$ & $T_{\mathrm{P} 2} /{ }^{\circ} \mathrm{C}$ & Residue (\%) \\
\hline PVC-DOTP & 264.4 & 310.1 & 300.9 & 465.0 & 13.16 \\
PVC-ESO & 283.1 & 337.4 & 310.6 & 460.8 & 10.89 \\
PVC-ECO & 280.3 & 340.1 & 307.9 & 465.3 & 11.08 \\
PVC-ACO & 268.3 & 326.9 & 302.4 & 459.0 & 11.83 \\
PVC-EACO & 275.7 & 334.8 & 309.6 & 465.5 & 10.22 \\
PVC-BCO & 268.8 & 322.2 & 301.7 & 457.8 & 11.47 \\
PVC-EBCO & 274.5 & 335.2 & 307.2 & 466.6 & 10.74
\end{tabular}



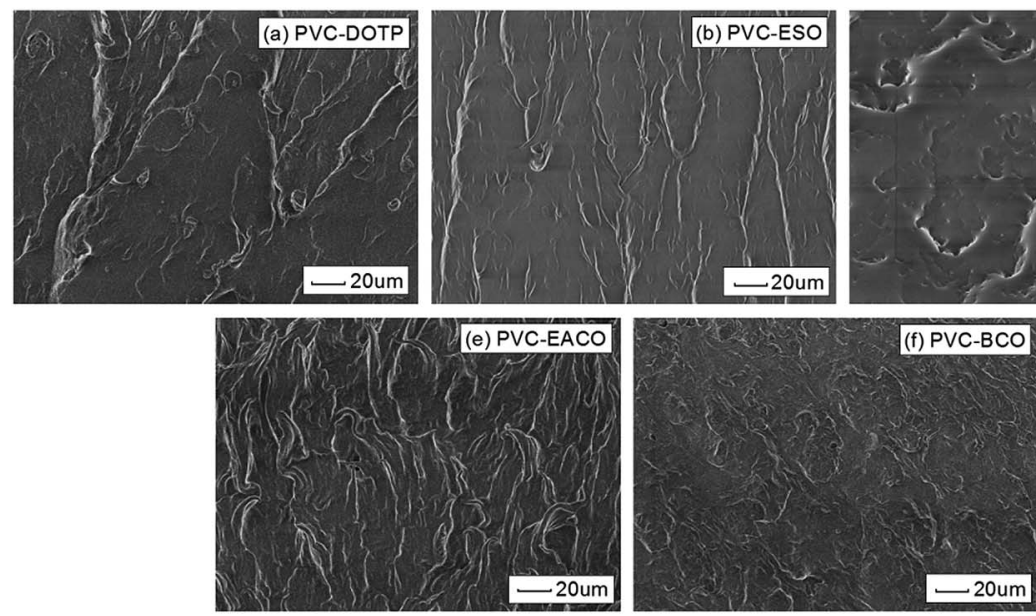
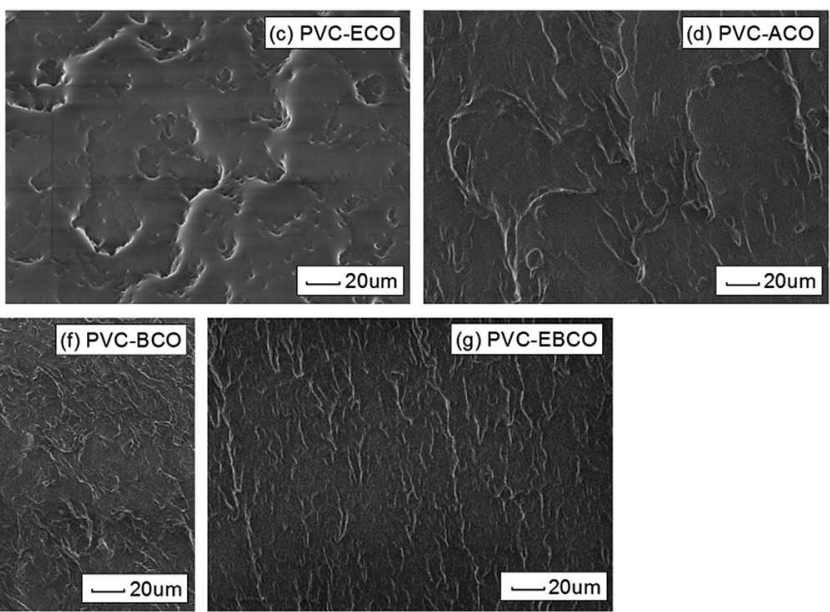

Fig. 11 SEM images of (a) PVC-DOTP, (b) PVC-ESO, (c) PVC-ECO, (d) PVC-ACO, (e) PVC-EACO, (f) PVC-BCO and (g) PVC-EBCO.

ECO did not have obvious protrusions and filaments, and the edge of the fracture was very smooth, which indicated that ECO did not show a good plasticizing effect with PVC. The section topography of PVC-EACO was much more complex than the others, and protrusions and filaments were more numerous and longer, which indicates that the plasticizer EACO had the best plasticizing effect, even better than that of DOTP and ESO.

\section{Conclusions}

In this study, five types of environment-friendly material plasticizers based on castor oil were synthesized. The synthetic plasticizers were added to PVC as the main plasticizer, and the properties of these PVC blends were investigated and compared to the commercial plasticizers DOTP and ESO. Tensile properties showed that the tensile strength and elongation at break of the PVC sample plastified by EACO were 18.5 and $10.0 \%$ higher than that of DOTP, and 13.9 and $23.8 \%$ higher than that of ESO, respectively. EACO also had generally better volatility, solvent extraction and migration stability properties than those of DOTP and ESO, and better than the other four plasticizers synthesized. All the tests illustrated that compared to hydroxy groups, carbon-carbon double bonds and phenyl groups, ester bonds, epoxy groups and alkyl groups can make plasticizers more compatible with PVC, showing better plasticizing effect and better thermal stability. Based on the above studies, the plasticizer EACO based on castor oil shows an excellent performance, in addition to this, during its synthesis, acetic acid produced by acetylation could be used in the subsequent epoxidation reaction to avoid excessive use of acid, and catalyst in the epoxidation reaction can be recycled. As an environmental-friendly plasticizer, it may have potential applications in medical equipment, children's toys, food packaging materials and other fields.

\section{Conflicts of interest}

There are no conflicts to declare.

\section{Acknowledgements}

The authors would like to thank the State Forestry Administration 948 project foundation for financial support under the Grant No 2015-4-55, China; and the Priority Academic Program Development of Jiangsu Higher Education Institutions (PAPD), China. The article is also supported by the Doctorate Fellowship Foundation of Nanjing Forestry University.

\section{Notes and references}

1 L. Giuseppe, D. F. Claudio and V. Alberto, Reprod. Toxicol., 2004, 19, 27-33.

2 G. Latini, Neonatology, 2000, 78, 269-276.

3 L. M. Kamendulis, J. S. Isenberg, J. H. Smith, G. Pugh Jr, A. W. Lington and J. E. Klaunig, J. Toxicol. Environ. Health, Part A, 2002, 65, 569-588.

4 L. M. De Espinosa and M. A. R. Meier, Eur. Polym. J., 2011, 47, 837-852.

5 P. G. Demertzis, K. A. Riganakos and K. Akrida, Eur. Polym. J., 1991, 27, 231-235.

6 P. G. Nihul, S. T. Mhaske and V. V. Shertukde, Iran. Polym. J., 2014, 23, 599-608.

7 M. A. Semsarzadeh, M. Mehrabzadeh and S. S. Arabshahi, Eur. Polym. J., 2002, 38, 351-358.

8 B. Badra and T. B. Mohamed, J. Appl. Polym. Sci., 2008, 107, 3442-3450.

9 O. Fenollar, N. L. Sanchez, S. D. Garcia, J. López and R. J. Balart, J. Mater. Sci., 2009, 44, 3702-3711.

10 B. M. Abdullah and J. Salimon, J. Appl. Sci., 2010, 1, 15431553.

11 P. Karmalm, T. Hjertberg, A. Jansson and R. Dahl, Polym. Degrad. Stab., 2009, 94, 2275-2281.

$12 \mathrm{M}$. Li, S. Li, J. Xia, C. Ding, M. Wang, L. Xu, X. Yang and K. Huang, Materials \& Design, 2017, 122, 366-375.

13 J. Chen, X. Li, Y. Wang, J. Huang, K. Li, X. Nie and J. Jiang, J. Appl. Polym. Sci., 2016, 133, 43668-43675.

14 J. Chen, X. Li, Y. Wang, J. Huang, K. Li, X. Nie and J. Jiang, Eur. J. Lipid Sci. Technol., 2017, 119, 1600216. 
15 W. Xu, X. Ge, L. Jin and R. Shao, Chem. Ind. Eng. Prog., 2015, 34, 1983-1988.

16 P. Jia, M. Zhang, L. Hu, G. Feng, C. Bo and Y. Zhou, ACS Sustainable Chem. Eng., 2015, 3, 2187-2193.

17 J. Chen, Z. Liu, X. Nie and J. Jiang, J. Mater. Sci., 2018, 53, 8909-8920.

18 P. Jia, M. Zhang, L. Hu, C. Bo and Y. Zhou, J. Therm. Anal. Calorim., 2015, 120, 1731-1740.

19 P. Jia, M. Zhang, C. Liu, L. Hu, G. Feng, C. Bo and Y. Zhou, RSC Adv., 2015, 5, 41169-41178.

20 P. Jia, M. Zhang, L. Hu, C. Liu, G. Feng, X. Yang, C. Bo and Y. Zhou, RSC Adv., 2015, 5, 76392-76400.

21 F. Wang, S. Pan, P. Zhang, H. Fan, Y. Chen and J. Yan, Fibers Polym., 2018, 19, 1057-1063.

22 J. Tan, T. Lu, J. Zhang, B. Xie, F. Zhu, M. Chen and X. Zhu, J. Taiwan Inst. Chem. Eng., 2018, 86, 18-24.

23 H. Zhang, F. Zhu, Y. Xu, X. Zhang and X. Zhu, Synth. Commun., 2017, 47, 486-495.
24 H. Zhang, D. Liu, T. Kang, Y. Wang, X. Zhang and X. Zhu, Chin. J. Org. Chem., 2016, 36, 1104-1110.

25 M. T. Rodríguez, S. J. García, R. Cabello and J. J. Suay, J. Coat. Technol. Res., 2005, 2, 557-564.

26 S. Lee, M. S. Park, J. Shin and Y. W. Kim, Polym. Degrad. Stab., 2018, 147, 1-11.

27 J. Chen, Z. Liu, J. Jiang, X. Nie, Y. Zhou and R. E. Murray, RSC Adv., 2015, 5, 56171-56180.

28 N. Lardjane and N. Belhaneche-Bensemra, J. Appl. Polym. Sci., 2009, 111, 525-531.

29 J. Kastner, D. G. Cooper, M. Marić, P. Dodd and V. Yargeau, Sci. Total Environ., 2012, 432, 357-364.

30 X. Wu, X. Zhang, S. Yang, H. Chen and D. Wang, J. Am. Oil Chem. Soc., 2000, 77, 561-563.

31 P. Karmalm, T. Hjertberg, A. Jansson and R. Dahl, Polym. Degrad. Stab., 2009, 94, 2275-2281.

32 H. Hosney, B. Nadiem, I. Ashour, I. Mustafa and A. EIShibiny, J. Appl. Polym. Sci., 2018, 135, 46270. 